\title{
VARIATIONS IN THE EXTENSOR MUSCLES OF THE HAND AND THEIR CLINICAL IMPLICATIONS
}

doi: 10.1590/S1807-59322008000400023

Srijit Das

I read with great interest the article entitled 'Anatomical variation of radial wrist extensor muscles: a study in cadavers' by Nayak et al., which was published in the 2008;63(1) issue of Clinics. ${ }^{1}$ The article had meticulous descriptions of some of the variations in the extensor muscles. The authors highlighted the clinical importance of the anomalous muscle in tendon transfer. It is of interest to know whether an anomalous muscle can be detected prior to any surgery so as to plan any tendon transfer accordingly. Such anomalous muscles are encountered during surgical procedures or routine anatomical dissections, and all such findings are incidental.

If there are additional muscles or tendons, the kinematics around the joint are certainly altered. An anomaly at any joint is likely to alter the biomechanics at that particular joint. It would be interesting to include any associated case histories, but these cannot be expected in cadaver studies. One must wonder if the excision of one of the anomalous' bellies or tendons would result in any change in muscle action. A detailed nerve supply to each of the muscles would have aided surgeons in planning the operations, although this study lacked this information. There are instances when the nerve may be compressed between the two heads of the muscle.

The measurements obtained in cadaveric studies are bound to differ from normal MRI or ultrasound measurements because there is a certain amount of shrinkage factor in the soft tissues after death. Could this shrinkage factor alter the measurement of strain? The strain on the muscle tendon as related to its fiber length has also been reported in the literature. ${ }^{2}$ The same study reported that the extensor carpi ulnaris muscle tendon has the smallest strain. ${ }^{2}$ Thus, anomalies pertaining to the extensor carpi ulnaris muscle tendon may influence the strain factor. The authors have measured the parameters in detail (shown in the table), which may be beneficial for planning and performing surgeries.

For palliative tendon transfer surgery for radial nerve palsy, researchers have highlighted the avoidance of radial deviation of the wrist by centralizing the insertion of the extensor carpi radialis longus. ${ }^{3}$ Thus, the extensor carpi radialis longus tendon plays an important role in muscle function.

Anatomists should be applauded for reporting such anomalies, but what really matters is whether normal anatomical measurements can be equated to the far superior ultrasound and MRI scans. Could ultrasound be used as a mandatory procedure for measuring different parameters related to the tendon before performing any surgery? A previous study used asymptomatic volunteers to assess different tendons. ${ }^{4}$ The same study described the importance of the variance in readings taken at different times and by different persons. ${ }^{4}$

Overall, it is an interesting article that could be beneficial for hand surgeons. The photographs are very clear, and the authors and the editorial board should be congratulated for this publication. 
Das S

\section{REFERENCES}

1. Nayak SR, Krishnamurthy A, Prabhu LV, Rai R, Ranade AV, Madhyastha S. Anatomical variation of radial wrist extensor muscles: a study in cadavers. Clinics. 2008;63:85-90.

2. Loren GJ, Lieber RL. Tendon biomechanical properties enhance human wrist muscle specialization. J Biomech. 1995;28:791-9.
3. Tubiana R. Problems and solutions in palliative tendon transfer surgery for radial nerve palsy. Tech Hand Up Extrem Surg. 2002;6:104-13.

4. O'Connor PJ, Grainger AJ, Morgan SR, Smith KL, Waterton JC, Nash AF. Ultrasound assessment of tendons in asymptomatic volunteers: a study of reproducibility. Eur Radiol. 2004;14:1968-73. 